\title{
Evaluation of Electron Temperature Fluctuations Using Two Different Probe Techniques in Plasma Assembly for Nonlinear Turbulence Analysis (PANTA)*)
}

\author{
Katsuyuki KAWASHIMA, Yasuhiro MIYOSHI, Stella OLDENBÜRGER ${ }^{1)}$, Shigeru INAGAKI ${ }^{1,2)}$, \\ Hiroyuki ARAKAWA, Yoshihiko NAGASHIMA ${ }^{1,3)}$, Takuma YAMADA ${ }^{1,3)}$, Makoto SASAKI ${ }^{1,2)}$, \\ Tatsuya KOBAYASHI, Kazuya URIU, Satoru SUGITA, Masatoshi YAGI ${ }^{1,2)}$, \\ Naohiro KASUYA ${ }^{1,4)}$, Akihide FUJISAWA ${ }^{1,2)}$, Sanae -I. ITOH ${ }^{1,2)}$ and Kimitaka ITOH ${ }^{1,4)}$ \\ Interdisciplinary Graduate School of Engineering Sciences, Kyushu University, Fukuoka 816-8580, Japan \\ ${ }^{1)}$ Itoh Research Center for Plasma Turbulence, Kyushu University, Fukuoka 816-8580, Japan \\ ${ }^{2)}$ Research Institute for Applied Mechanics, Kyushu University, Fukuoka 816-8580, Japan \\ ${ }^{3)}$ Graduate School of Frontier Sciences, The University of Tokyo, Tokyo 277-8561, Japan \\ ${ }^{4)}$ National Institute for Fusion Science, Toki 509-5292, Japan
}

(Received 7 December 2010 / Accepted 27 May 2011)

\begin{abstract}
Electron temperature fluctuations are measured with a rotatable triple probe in PANTA. Effects of fluctuation phase are cancelled by aligning the probe pins in the propagation direction of fluctuations. Evaluated electron temperature fluctuations normalized by average electron temperature are much smaller than normalized density fluctuations and normalized floating potential fluctuations in low electron temperature PANTA plasma. Additionally conditional sampling technique is applied to a single probe measurement.
\end{abstract}

(C) 2011 The Japan Society of Plasma Science and Nuclear Fusion Research

Keywords: electron temperature fluctuations, triple probe, conditional sampling

DOI: $10.1585 /$ pfr.6.2406118

\section{Introduction}

Turbulent fluctuations are widely accepted as a major contributor to the cross-field transport in magnetically confined plasmas. Correlated fluctuations of density, poloidal electric field and temperature result in time-average flux. Simultaneous measurement of these fluctuations with high spatial and temporal resolutions is required for experimental investigation of this fluctuation-driven transport. Langmuir probe diagnostics are suitable to obtain highly resolved measurements of electric field and density fluctuations. However real-time data of the electron temperature fluctuations are more difficult to obtain and therefore, they have often been neglected in previous research on fluctuation-driven transport, following the assumption that the fluctuation level is sufficiently low. In order to support this assumption a careful estimation of fluctuation level would be necessary. Unfortunately, methods to estimate electron temperature fluctuations from fast-sweeping current-voltage characteristics of a single probe suffer from large error due to capacitive current when the sweeping frequency of probe bias approaches the frequencies relevant for temperature fluctuations. The alternative triple probe method is affected by a large error due to phase delay of fluctuations or shadowing between pins [1,2]. The exis-

author's e-mail: kawashima@riam.kyushu-u.ac.jp

*) This article is based on the presentation at the 20th International Toki Conference (ITC20). tence of temperature fluctuations has been indicated in several basic plasma experimental devices, including Large Mirror Device-Upgrade (LMD-U) linear plasma [3] and Tokyo Spherical Tokamak-2 (TST-2) tokamak plasma [4]. In this paper, we evaluate the level of temperature fluctuations in the linear plasma of the Plasma Assembly for Nonlinear Turbulence Analysis (PANTA), using two different improved probe techniques. On one hand, for the triple probe, the pins are carefully aligned to the fluctuation structure prior to measurement. On the other hand, single probe measurements are analyzed using a conditional sampling method, which does not require a fast sweeping of the bias voltage. Estimation of temperature fluctuations by two different techniques makes it possible to crosscheck the results and increases the confidence.

\section{Experimental Setup}

Fluctuation measurements of electron temperature were carried out in PANTA. Figure 1 shows a top view of the PANTA experiment and arrangement of probes. PANTA is a linear magnetized device and produces a cylindrical plasmas (diameter $0.1 \mathrm{~m}$, length $4 \mathrm{~m}$ ) with a helicon wave $(7 \mathrm{MHz}, 3 \mathrm{~kW})$ using a double-loop antenna around a quartz tube. The quartz tube is filled with argon gas with a pressure of $0.4 \mathrm{~Pa}$. The magnetic field is $0.09 \mathrm{~T}$. The vacuum vessel is at ground potential. The endplate is directly 

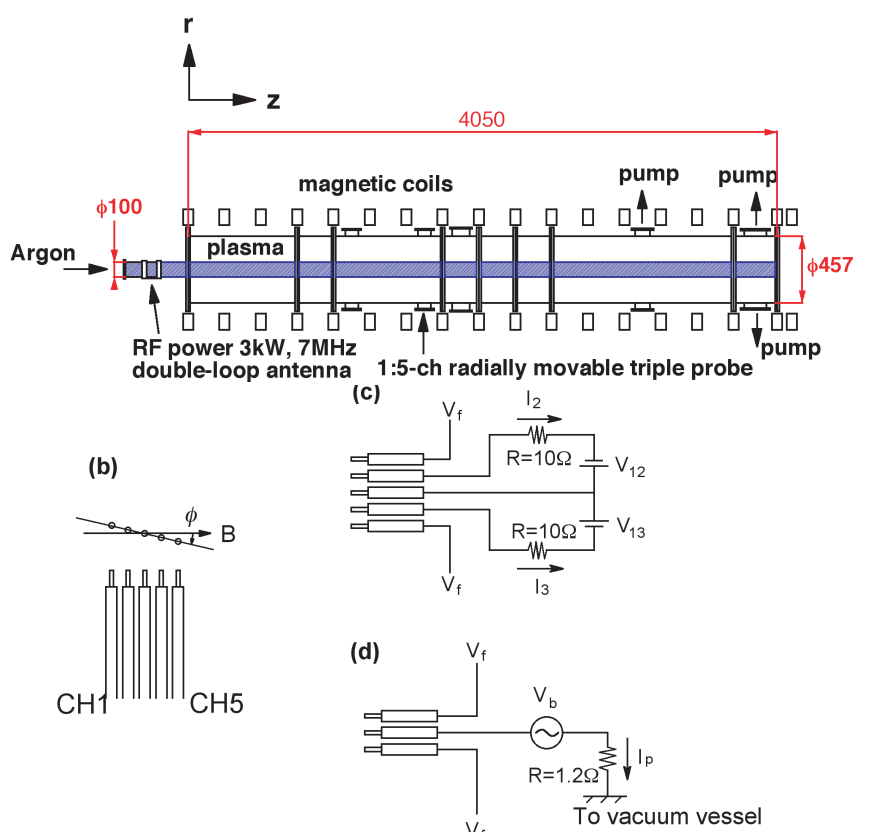

Fig. 1 Schematic view of the Plasma Assembly for Nonlinear Turbulence Analysis (PANTA) (a), the tip of the 5channel triple probe array (b) and the circuit used for the two methods (c), (d). The 5-channel triple probe is rotated until fluctuations on the different pins are in phase.

connected to the vacuum vessel. The Debye length is estimated to be about $6 \mu \mathrm{m}$ in this experiment. Evaluation of electron temperature fluctuations was carried out using the same 5-channel probe in two different configurations. The 5-channel probe consists of five tungsten pins with a diameter of $0.7 \mathrm{~mm}$ and a length of $3 \mathrm{~mm}$ arranged on a line and separated by $3 \mathrm{~mm}$. This probe is allowed to rotate and move radially thanks to a probe shaft sealed by an O-ring. By aligning the probe pins with the axial direction of the fluctuating structure, the phase delay between the pins can be canceled. The rotation angle $\phi$ relatively to the magnetic field line is positive in the clockwise direction. The left edge probe is defined as channel 1 , and the right edge one is defined as channel 5. This probe is installed at $z=$ $1.675 \mathrm{~m}$ from the quartz tube. Figure 1 (c) shows the circuit for the triple probe method. In this case, the electron temperature is calculated from the plasma currents that appear when a voltage is applied between the center pin and the side pins. The relation between plasma current and electron temperature $T_{\mathrm{e}}$ is given as [5]:

$$
\frac{2 I_{2}+I_{3}}{I_{2}+2 I_{3}}=\frac{1-\exp \left(-e V_{12} / T_{\mathrm{e}}\right)}{1-\exp \left(-e V_{13} / T_{\mathrm{e}}\right)}
$$

In addition, the relation between plasma current and electron density $N_{\mathrm{e}}$ is given as [5]:

$$
\begin{array}{r}
I_{\text {is }}=\frac{I_{3}-I_{2} \exp \left(-e\left(V_{13}-V_{12}\right) / T_{\mathrm{e}}\right)}{1-\exp \left(-e\left(V_{13}-V_{12}\right) / T_{\mathrm{e}}\right)} \\
=0.61 N_{\mathrm{e}} e S \sqrt{T_{\mathrm{e}} / m_{\mathrm{i}}},
\end{array}
$$

where $S$ is the probe pin surface, $e$ is elementary charge and $m_{\mathrm{i}}$ is ion mass. In this experiment, $V_{12}$ is $2 \mathrm{~V}, V_{13}$ is $10 \mathrm{~V}$ and the resistance is $10 \Omega$. The inner three pins of the 5-channel probe are used to obtain $I_{2}$ and $I_{3}$, while the outer two pins measure floating potential. Figure 1 (d) shows the circuit connected to the 5-channel probe for the single probe method. In that case, the center pin measures plasma current while a low frequency voltage sweep is applied between the pin and the vacuum vessel. The other pins measure floating potential. The resistance used to measure plasma current is $1.2 \Omega$. The sweeping bias is $100 \mathrm{~V}$ peak-to-peak and sweep frequency is $100 \mathrm{~Hz}$. The floating potential at the position of the center pin is estimated from the average of potentials measured by neighboring pins and used as reference for the conditional sampling. The global range of floating potential values is separated into six regions using mean value and standard deviation. Depending on the instantaneous value of floating potential, current and voltage data points are redistributed to form six distinct characteristics. Each characteristic corresponds to one range of floating potential. The electron temperatures are calculated for each characteristic. That way it is possible to investigate correlation between electron temperature and floating potential. The current-voltage curve for each floating potential region could be studied.

\section{Results of Triple Probe Measure- ments}

Although the probe is smaller than the fluctuation structure scale, the probe had to be aligned with the direction of the fluctuation structure to cancel possible phase delay before we measured the electron temperature by using the triple probe method. Phase matching has been tested for several positions between $-5^{\circ}$ to $+15^{\circ}$. The phase of fluctuations showed the best match at an angle of $\phi=\phi_{m}=3^{\circ} \pm 1.5^{\circ}$ at $r=4 \mathrm{~cm}$. This situation is shown in Fig. 2. Figure 2 (a) shows time series of potential on all channels of the probe. The phase delay between the channels is very small. The cross phase between both edge channels has been calculated and the result is shown in Fig. 2 (b). The phase delay is about 0.01 radian at around $1 \mathrm{kHz}$, which is the typical mode frequency in PANTA (as is shown in Fig. 5). This indicates that with this alignment phases of fluctuations almost match for a typical mode. The azimuthal wavelength and axial wavelength of a typical mode are $0.24 \mathrm{~m}$ and $4 \mathrm{~m}$, respectively. Observed $\phi_{m}$ is in agreement with that estimated for a typical mode structure $\left(3.6^{\circ}\right)$. After completing the alignment procedure, the triple probe circuit illustrated in Fig. 1 (c) was used to record plasma fluctuations. Figure 3 shows the obtained radial profiles of mean floating potential, mean electron temperature and mean electron density according to Eq. 1 and Eq. 2. Error bars correspond to the standard deviation of fluctuations. The floating potential increases between $r=$ $3 \mathrm{~cm}$ and $r=4 \mathrm{~cm}$, while the electron density decreases. The electron temperature is almost flat over the whole profile. Figure 4 shows the time evolutions of fluctuations 

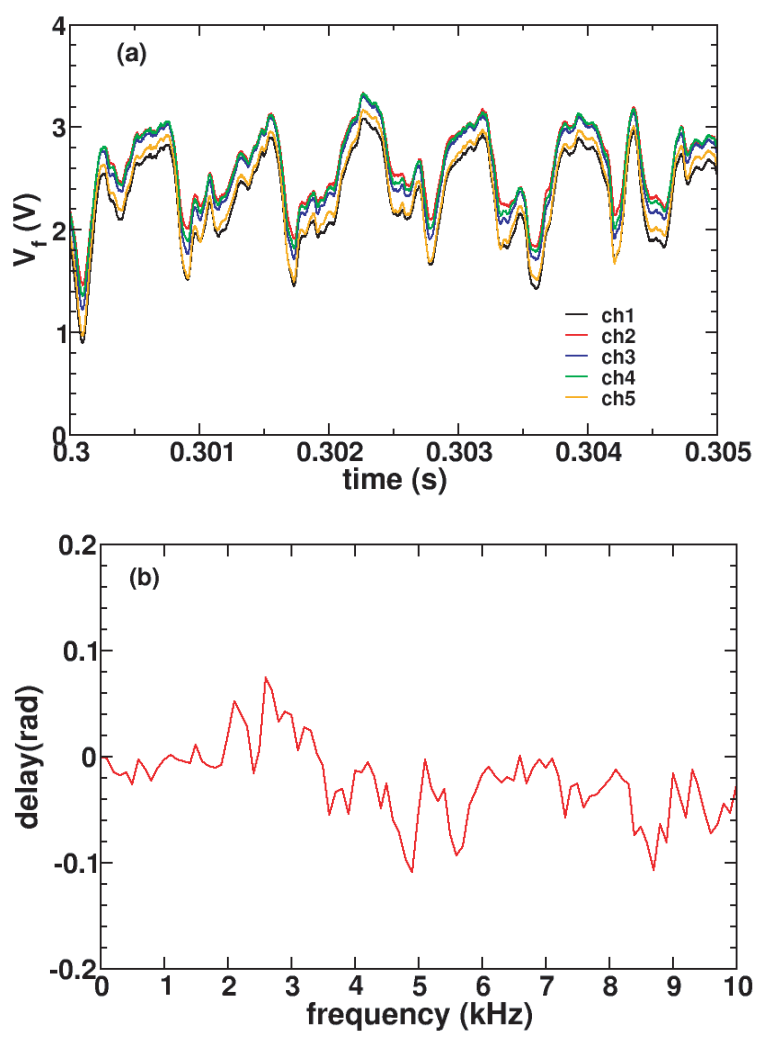

Fig. 2 Typical time evolutions of floating potential measured by the 5-channel triple probe array (a) and cross phase delay between channel 1 and channel 5 of the probe array (b).

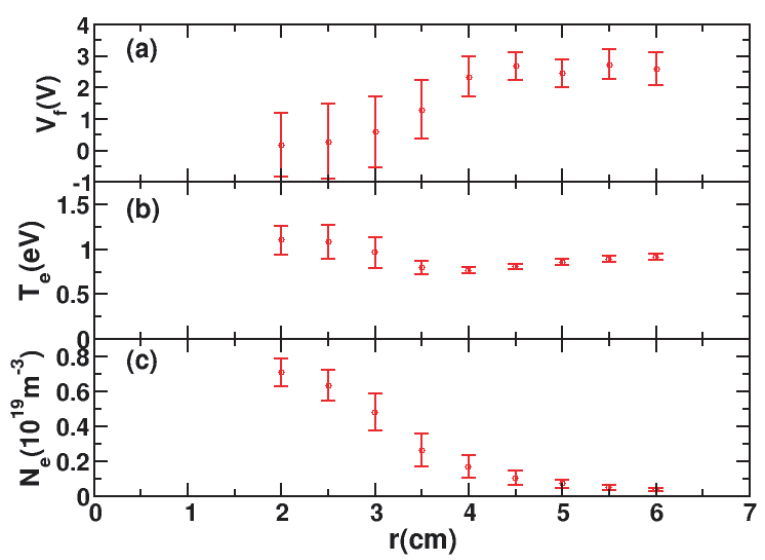

Fig. 3 Radial profile of mean floating potential (a), mean electron temperature (b), mean electron density (c). Standard deviation is shown as error bars.

at $r=4 \mathrm{~cm}$. Electron temperature fluctuations resemble square waves, not monochromatic waves in Fig. 4, and thus electron temperature fluctuations consist of a fundamental mode and higher harmonic modes. Figure 5 shows power spectra of normalized fluctuations at $r=4 \mathrm{~cm}$. Floating potential and electron temperature have been normalized by average electron temperature and electron density has been normalized by average electron density. The fluctuations have similar characteristics in the low frequency

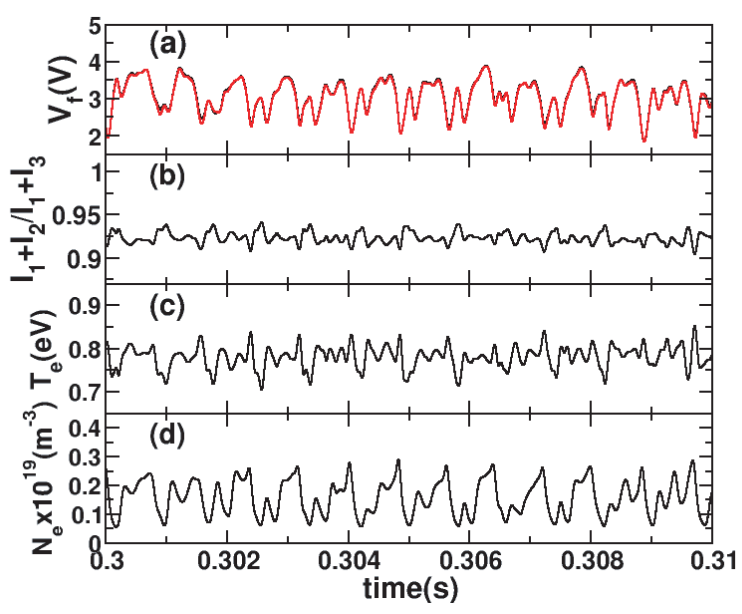

Fig. 4 Time evolutions of floating potential of channel 1 (black line) and channel 5 (red line) (a), calculated current ratio from $I_{2}$ and $I_{3}$ (b), electron temperature (c) and electron density (d).

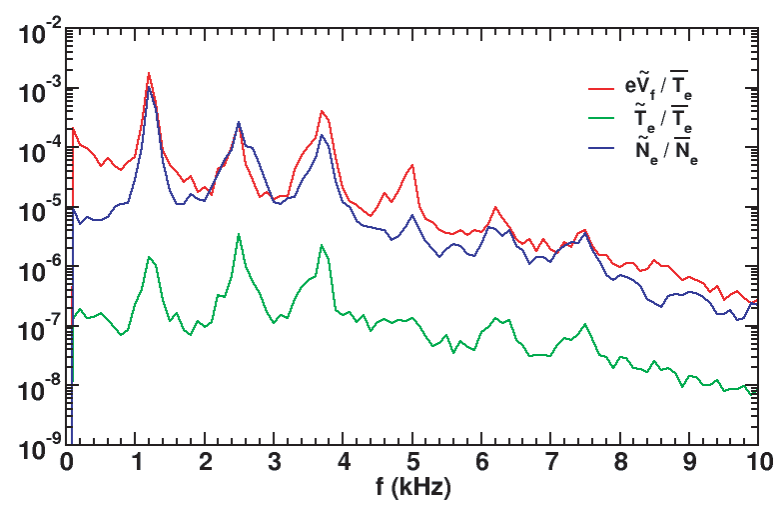

Fig. 5 Power spectrum of normalized fluctuation data. Red line corresponds to normalized floating potential fluctuations, blue line to normalized electron temperature fluctuations and green line to normalized electron density fluctuations.

range. Normalized fluctuation amplitudes of electron density and floating potential are predicted to be comparable by virtue of the Boltzmann relation, but a small difference exists. The comparison between fluctuation amplitudes of electron temperature and electron density, yields a ratio of approximately $1 / 12$. Temperature fluctuations are small compared to the amplitude of floating potential fluctuations. This small value of $\left(\tilde{T}_{\mathrm{e}} / T_{\mathrm{e}}\right)\left(\tilde{N}_{\mathrm{e}} / N_{\mathrm{e}}\right)^{-1}$ has been expected, because the temperature gradient is much weaker than the density gradient as is shown in Fig. 3 [6]. In addition, while the fluctuations of floating potential and electron density are highest at the fundamental mode (approximately $1 \mathrm{kHz}$ ), the fluctuations of electron temperature are highest at the second harmonics (approximately $2.5 \mathrm{kHz}$ ). The difference between the ratio of $\left(\tilde{T}_{\mathrm{e}} / T_{\mathrm{e}}\right)\left(\tilde{N}_{\mathrm{e}} / N_{\mathrm{e}}\right)^{-1}$ at $f$ $=1.2 \mathrm{kHz}$ and at $f=2.5 \mathrm{kHz}$ demands further investigations. 


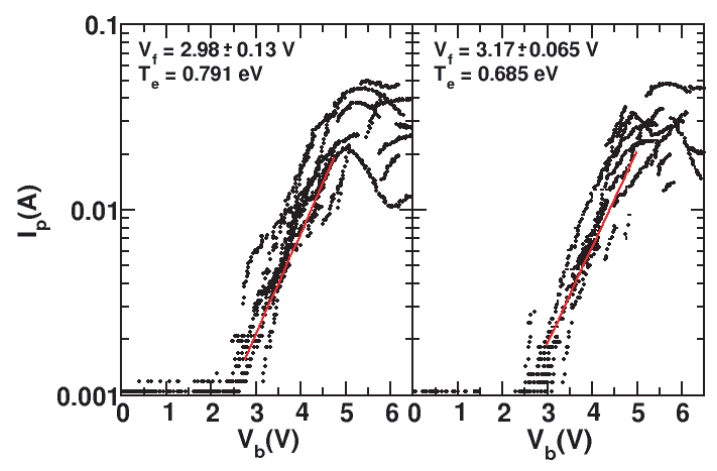

Fig. 6 Fitting of current-voltage curves and obtained electron temperatures for each range of floating potential.

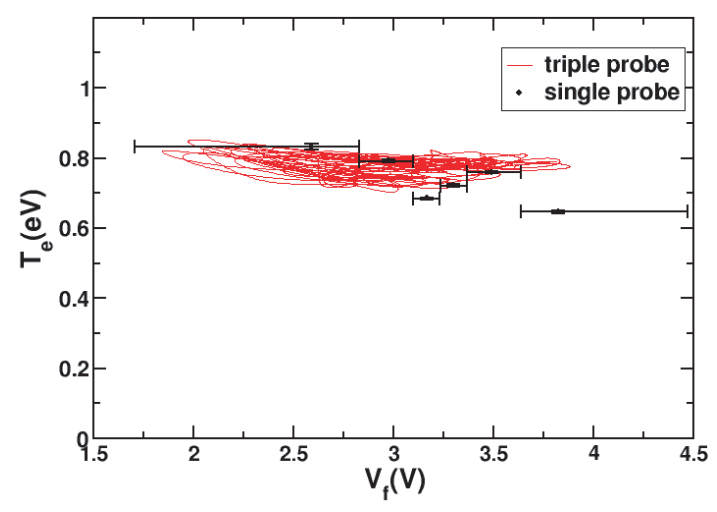

Fig. 7 Relation between electron temperature and floating potential. The results obtained by using the triple probe are compared with those obtained by using the single probe. Both measurements were carried out at a radial position of $4 \mathrm{~cm}$. They were carried out on two successive discharges with the same discharge conditions.

\section{Conditional Sampling Technique}

In this section, we present the results of the conditional sampling technique based on floating potential fluctuations with a single probe at $r=4 \mathrm{~cm}$. The floating potential measured on the both side pins (see Fig. $1(\mathrm{~d})$ ) is separated into six levels. The current-voltage curve of the single probe is discriminated with respect to each floating voltage level. Figure 6 shows the typical current-voltage characteristics for two floating potential levels where $V_{\mathrm{b}}$ is bias sweeping voltage. The floating potential and its range are indicated. In the figure, plasma current is plotted in logarithmic axis and sweeping voltage is plotted in linear scale. Electron temperatures were evaluated from each current-voltage curve. The figures show that the currentvoltage curve is shifted in the voltage axis depending on floating potential. Figure 7 summarizes the resulting electron temperature for different floating potentials. The obtained mean electron temperatures are almost the same for the single probe and the triple probe. Electron temperature and floating potential show weak negative correlation for the triple probe as well as for the single probe. In order to obtain quantitative understanding further investigations are required, since one has to take into account the phase relation between them.

\section{Discussion and Summary}

We measured radial profiles of floating potential, density and temperature by using an improved triple probe, whose pins were aligned taking into account the propagations of the fluctuations. This probe also provided realtime measurements of plasma fluctuations, including electron temperature fluctuations. The results show that electron temperature fluctuations normalized by mean temperature are small compared to normalized electron density fluctuations and normalized floating potential fluctuations. These results support the working hypothesis that most of the ion saturation current fluctuation and the floating potential fluctuation come from fluctuations of density and space potential, respectively.

\section{Acknowledments}

This work is partly supported by a grant-aid for scientific research (S) from JSPS(21224014), the grant-in-aid for Scientific Mechanics Kyushu University and National Institute for Fusion Sciences (NIFS10KOAP023).

[1] S.L. Chen and T. Sekiguchi, J. Appl. Phys. 36, 2363 (1965).

[2] C. Silva et al., Am. Phys. 75, 4314 (2004).

[3] S. Inagaki et al., Annual meeting on JSPF 28aB14P.

[4] Y. Nagashima et al., Plasma Fusion Res. Special Issue in press.

[5] H. Lin et al., Am. Phys. 63, 4611 (1992).

[6] W. Horton, Review of Modern Phys. 71, 3 (1999). 